\title{
ANTROPOLOGÍA
}

\section{El cerro y el felino. Un caso de unidad de grupo en la mitología en los Andes y la Amazonía}

\section{RESUMEN}

El presente artículo constituye un ejercicio comparativo de las representaciones de los campesinos quechua de los andes centrales con aquellas de los pueblos del piedemonte oriental de los andes centrales con especial referencia a los ashaninka y con las de otros pueblos indígenas de las bajas tierras como los desana tukano en lo referente a la imagen de los cerros sagrados vinculados a los felinos considerados protectores de las presas de caza.

PALABRAS CLAVE: Tierras bajas de América del sur- Andes-Montañas sagradas- presas de caza

\section{The mountain and the feline. A case of group unity in Andean and Amazonian mythology}

ABSTRACT

This article is a comparison between the representations of the quechua peasents of the central Andes and the preandean arawak ashaninka as well as other peoples of lowland south america like the desana tukano about sacred mountains and their relations with preys and the felines of the region seen as their guardians.

KEYWORDS: Lowland South America- Andes- Sacred mountains- preys 


\section{Introducción}

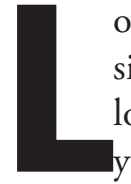

os mitos y los ritos forman parte de los sistemas simbólicos de un pueblo. Estos se acumulan a lo largo de su historia de acuerdo a los contactos relaciones con otros pueblos, relaciones que pueden ser de guerra o intercambio, sometimiento o lucha por la autonomía (Dumézil 2000) lo que es el caso de los cazadores-recolectores campa ashaninka, descendientes de los anti de las bajas tierras amazónica. La etnografía de las últimas décadas ha mostrado cómo los ashaninka se han encontrado bajo el influjo de sus vecinos de las zonas adyacentes tanto los quechua de las altas tierras de los andes como las poblaciones de las bajas tierras amazónicas. Muestra del influjo andino son los mitos de pachakama e Inka reportados por Varese (1973) y Weiss (1975) y Fernández (1984, 1987). En el presente artículo nos proponemos complementar esta imagen con un mito compartido tanto por las poblaciones ashaninka de las bajas tierras de la Amazonia como por los quechua de los andes al que hasta el momento los etnógrafos no han prestado atención, aquel de la figura de las montañas sagradas donadoras de animales custodiadas por los grandes felinos, representación atestiguada en el registro etnográfico de sus vecinos quechua del área andina con quienes han tenido una larga historia de relación a lo largo del sector que ocupan en la frontera ecológica y cultural que separa ambas poblaciones.

El gran etnógrafo Alfred Métraux ya hacía notar que las poblaciones nativas de la Amazonía «atribuyen a las especies animales un 'dueño', (...) que cuida por su multiplicación e impide su destrucción inútil» (Métraux 1962: 125). Posteriormente ReichelDolmatoff $(1968,1970)$ observó para los Desana del Vaupés colombiano esta misma figura mitológica, Waí- maxsë a la que se atribuye como lugar de habitación cerros aislados cubiertos de grietas donde habitan rodeados de sus animales (1968: 59). Waimaxsë cuida allí de sus animales y se preocupa en especial por la multiplicación de venados y tapires aunque a veces puede salir a cazarlos con una varilla. El jaguar se identifica con el payé o chamán (1968: 74). Los chamanes son los únicos que pueden visitar estos lugares para negociar con Vai-maxcë el envío de presas de caza a los cazadores. La figura del «dueño de los animales» está bastante extendida en la Amazonia presentándose entre los mai huna (Bellier 1994: 134); los yagua (Chaumeil 1994: 283); los tikuna (Goulard
1994: 387); los shipibo-conibo (Morin 1998: 370); y los candoshi (Surrallés 2007). A este caso se añade el de la población ashaninka que comparte la creencia de que el dueño de los animales es un espíritu que habita en lo alto del cerro que domina una región donde los jaguares contribuyen a cuidar a las presas.

\section{Conceptos y métodos empleados}

El tema que nos ocupa requiere de la explicación de lo que se entiende por sistema simbólico. Este término califica los fenómenos culturales que tienen por característica estar provistos de un valor percibido como inmediatamente expresivo: mitos, ritos, creencias, etc. Estos reorganizan la experiencia sensible al interior de un sistema semántico (Lévi-Strauss 1958). Dentro del sistema simbólico el mito está constituido en base a metáforas que redescriben lo que es, y de allí que se diga que existe una forma de pensamiento mítico caracterizado por razonamientos de tipo analógico (Barthes 1985). En cuanto al ritual, este es actos no verbales y la estructura lingüística de las palabras sagradas que los acompañan (Tambiah 1990: 184). Siendo las sociedades en cuestión de cazadores-recolectores y campesinos entenderemos aquí por lo sagrado una noción de fuerza exuberante y fecundante capaz de traer a la vida y de hacer surgir las producciones de la naturaleza;aquello animado de un poder divino y que está prohibido de contacto con los hombres (Benveniste 1969 vol. II). En esta investigación usaremos también el concepto de función, definido por Dumezil como el tipo de acciones atribuidas a una divinidad o un héroe. Un personaje mítico se define por el tipo de acción que se le atribuye lleva a cabo en el sistema simbólico, lo que lo distingue del resto de divinidades en dicho sistema. En este sentido, el análisis de un determinado personaje mítico o divinidad en un sistema aislado del conjunto de los otros personajes que forman parte de dicho sistema carece de sentido. Un personaje mítico solo puede ser analizado en sus relaciones con los otros personajes que forman parte de dicho sistema. Concepto estrechamente ligado al de función es el de desdoblamiento desarrollado por LéviStrauss (1991). Este concepto alude al hecho de que en un mismo o diferentes sistemas, un mismo personaje mítico definido por sus funciones específicas puede presentarse bajo otro nombre. Dicho de otra manera, divinidades o héroes con diferentes nombres pero con la misma función son en realidad el mismo. Finalmente 
haremos uso del concepto de grupo de transformaciones para designar «las correlaciones formales que mantienen entre sí todas las variantes conocidas o desconocidas de un determinado mito»; Greimas y Courtés 1982; ver también Sperber 1971). El tema tratado nos lleva a comparar en base a la batería de conceptos presentada informaciones etnográficas publicadas tanto andinas como amazónicas, en especial de los ashaninka registradas por el autor de acuerdo al método de análisis estructural desarrollado por Lévi-Strauss y sus seguidores.

\section{Antecedentes históricos}

Los ashaninka pertenecen a la rama de las poblaciones de lengua arawak que los lingüistas llaman los protomaipureanos. Originalmente se encontraban, hace 2500 años en el curso medio del Amazonas. Mas tarde estas poblaciones fueron empujadas fuera de esta zona de ricos recursos de caza y pesca por poblaciones de lengua tupí. Un sector de estas poblaciones se encaminó río arriba llegando al río Ucayali donde se ubicó por un tiempo. Posteriormente continuaron su marcha hacia el sur expulsados del Ucayali por poblaciones de lengua pano. En esta dirección llegó al colinoso territorio de la frontera ecológica entre la cuenca amazónica y las altas tierras de los Andes centrales, deviniendo los campa ashaninka encontrados por los conquistadores europeos (Noble Cook 1965; Lathrap 1970). Ellos devendrían la sociedad ashaninka, que posteriormente atravesó un proceso de sedentarización de sus miembros en aldeas cada vez más independientes así como también una pérdida de sus mecanismos integrativos.

Originalmente organizados en pequeños establecimientos de no más de cinco familias esta extrema dispersión contrastaba con un extendido sistema de intercambios que tenía por eje la sal gema obtenida de diferentes yacimientos en el territorio del grupo. Este mecanismo hacía que se produjera valores sociales allí donde la guerra intra-tribal era común.

Los estados andinos prehispánicos Huari e Inka intentaron en algún momento establecer un control directo de la población arawak en la zona del bajo Apurimac y las fuentes del río Perené para controlar la producción de hoja de coca (Bonavia y Ravines 1967; Parsons y Hastings 1982; Renard-Casevitz et al. 1986; Raymond 1982, 1992). La motivación principal de los gobernantes andinos para entrar a la zona había sido acceder a cantidades mayores de hoja de coca, lo que en el modelo andino de reciprocidades asimétricas entre el Estado y los campesinos, les permitía movilizar una cantidad mayor de mano de obra agrícola en las altas tierras. La extrema dispersión de los arawak impidió toda sumisión a estos estados.

Con el contacto europeo, habiendo aceptado inicialmente la presencia de los misioneros católicos que les proveyeron de la tecnología del hierro introduciendo los medios para la explotación de yacimientos de este mineral en su territorio, estos fueron luego expulsados debido a las múltiples epidemias desatadas en los pueblos de misión en que eran concentrados. La rebelión de Juan Santos en 1742 marca el punto más alto del rechazo a los extranjeros (Santos Granero 1988).

La resistencia de los pueblos arawak del piedemonte terminaría hacia 1883 debido en gran medida a las epidemias que golpearon duramente a estas poblaciones por ese entonces. El Ejercito Peruano empieza un largo proceso de ocupación del territorio y colonización.

Hacia 1887 con el boom del caucho se produce una ola de violencia por la adquisición de mano de obra indígena esclava por los patrones. En este periodo se produjo la transformación de las bases de la reproducción material de la sociedad ashaninka y la sedentarización de la población indígena en aldeas. Los útiles cortantes en piedra, madera y hueso habiendo sido reemplazados y sus técnicas de producción perdidas, las actividades agrícolas, así como la producción de los útiles empleados en la caza, la pesca, la transformación de alimentos y la producción de vestimentas - en suma, las condiciones materiales de vida- no podían ser reproducidas ya sin la participación de útiles de acero producidos industrialmente. Así a partir de esa época, la reproducción de las condiciones materiales de existencia de la sociedad ashaninka pasó a depender de las relaciones de intercambio con la sociedad peruana. Esta situación se afianzó en los años 1940 con la demanda de caucho debido a la guerra mundial y posteriormente en los años 1950 por la expansión del mercado mundial, a la que no estuvo exenta la región ocupada por los ashaninka, (Barclay y Santos 1995).

Misioneros adventistas cuya llegada hacia 1950 fue interpretada como la venida de una nueva era desde una concepción mesiánica del mundo, llevó a la formación de grandes aldeas y contribuyó a la sedentarización de la población ashaninka en nuevos pueblos. Estos se dispersaron con el estallido de nuevas epidemias resultado de la concentración poblacional excesiva 
(Bodley 1972). Sin embargo nuevas aldeas se formaron por la intervención de los misioneros evangélicos del Instituto Lingüístico de Verano. Los fundadores de las actuales comunidades indígenas son huérfanos supervivientes de todos estos violentos procesos. Estos se detendrían con la promulgación en 1974 de la Ley de Comunidades Nativas y Campesinas que impulsó el proceso final de sedentarización de la población en aldeas (Rojas Zolezzi 2014).

\section{El cerro y los no humanos en los Andes}

Es Gary Urton (1985) quien desarrolla el estudio de los no humanos y su vinculación con el cerro entre los campesinos y ganaderos andinos del Cuzco con profundidad. Con anterioridad a este autor Arguedas (2013) había recogido mitos explicativos de la orografía de ciertas regiones, Valderrama y Escalante (1988, 1997) el simbolismo de la relación entre la agricultura y el cerro como donador de agua y Ansión mitos vinculados a la actividad del arrieraje y al poder y el liderazgo en la comunidad (Ansión 1988).

Los quechua, agricultores y ganaderos denominan apus a las montañas más altas de la región donde viven y son consideradas sagradas. En sus cumbres se cree habitan los espíritus llamados aukis. Hay no humanos que se considera pertenecen a los apus y los humanos pueden hacer uso de ellos pero no les pertenecen. Es el caso de los venados y cierto tipo de ovejas. Los venados (Hippocamelus antisensis) son llamados según Urton «vacas de los apus» y para comerlo un humano debe hacer un "despacho" al apu por dicho animal. Un despacho es una colección de hojas de coca (kintu) que es quemado o enterrado en la cima del cerro como una forma de gesto propiciatorio para que los apus actúen en favor de los humanos.

Una relación similar se establece entre el apu y ciertos animales domesticados. Así, un hombre puede enterrar un despacho cerca de un manantial u ojo de agua en las alturas los que se considera son lugares de origen de ovejas y camélidos bebé de los cuales ellos emergen. Estos animales son considerados especialmente fecundos y el que los encuentra puede quedárselos y formar con ellos un rebaño ya que paren crías todos los años.

Los pumas (Puma concolor) son considerados «hijos de la tierra" y altomisayoq, es decir chamanes y son capaces de comunicarse con los espíritus de la tierra y el cerro. Si el puma llega a saber que alguien habla en contra de los espíritus, van a la casa del ofensor de noche y toman uno de sus animales (Urton 1985).

\section{El cerro y los no humanos entre los ashaninka}

Entre los cazadores recolectores ashaninka del piedemonte oriental de los andes centrales, cuando las presas no vienen más, lo que es atribuido a la conducta individual de algún cazador, un chamán puede ir a visitar al «dueño de los animales», el que es descrito como un ser antropomorfo muy delgado ; la negociación con este ser tiene en buena cuenta el carácter de una súplica, ya que los términos atribuidos al chamán vendrían a ser los siguientes: «Para que mi hijo que tiene hambre,que no tiene nada que comer, envíame animales que cazar». Por medio de las visiones del ayahuasca (banisteriopsis caapi) el chamán puede viajar volando hasta donde este maninkari o divinidad habita, en las cumbres de los cerros que dominan el territorio de la comunidad lo$\mathrm{cal}$, donde se dice que las presas se encuentran dentro de corrales bajo el cuidado de su dueño mítico. Las analogías para describir estos lugares son múltiples: de una parte la imagen del ganado en corrales presentada por los vecinos quechua, de otro lado el pecari (tayassu pecari), uno de los escasos mamíferos terrestres que se desplaza en grandes bandas; los árboles en época de fructificación, en cuyas copas diferentes especies de aves encuentran alimento, son también el punto de partida para desarrollar esta figura entre estos cazadores recolectores. Según los chamanes entrevistados cuando el chamán llega a la casa de la dueña de los pecaríes - ser femenino de manera excepcional en esta subcategoría de seres masculinos - sopla sobre una piel de este animal, produciendo la multiplicación de este tipo de presa, figura anteriormente reportada por Weiss (1975) y luego por Rojas Zolezzi (2004, 2014). Hvalkof y Weber (2005: 256) reportan que el nombre de los dueños de los animales es nantatziri en la zona del gran pajonal y Rojas Zolezzi el de iriri éntre los ashaninka que habitan en los grandes rìos (Rojas Zolezzi 2014). Luego, el cháman debe llevar estas presas al territorio habitado por los cazadores. El chamán, de acuerdo a nuestros informantes, transformado en colibrí debe hacer un viaje provisto de un morral grande y resistente para cargar con todas las presas. Los vientos fuertes anuncian el retorno del colibrí con su preciada carga. Dentro del ciclo anual, los colibríes retornan al 
territorio ashaninka cuando las lluvias han terminado, en la estación seca (mayo-agosto), época de la caza por excelencia y aquella de la aparición entre las cabañas de las plantas de tabaco, cuya semilla, se dice es traída por estos. Así, esta coincidencia en el ciclo anual constituye un punto de partida para establecer una homología entre el chaman y el colibrí por la cual se atribuye al chamán el poder de transformarse en este ser con valor de intermediario con los espíritus de los cerros (Rojas Zolezzi 2014: 275).

Un chamán, al momento de morir, es llevado a la cumbre más alta dentro del territorio del grupo local, donde su cuerpo es abandonado sin ser enterrado. Se dice que los chamanes más sabios podrán entonces entrar al cielo henoki y sumarse a los maninkari que allí habitan. Un chamán menos sabio se transformará en un jaguar (Panthera onca), el cual estará asociado a dicha montaña. En el colinoso territorio ashaninka, en cada región existen determinados cerros más altos que otros, en los cuales se reúnen de vez en cuando los chamanes fallecidos transformados en jaguares protectores de los diferentes grupos locales. Estos lugares son zonas cuyo acceso está prohibido a los cazadores y en los que las presas se reproducen (Rojas Zolezzi 2014: 295) .

\section{Una unidad de grupo}

Podemos encontrar que el mito desana del «dueño de los animales» habitante del cerro y el mito ashaninka del «dueño de los animales» son simétricos respecto al quechua del origen de las ovejas y auquénidos bebé que emergen de los manantiales del cerro, como podemos ver en el siguiente esquema en que presentamos las correlaciones entre estos tres segmentos textuales.

Estos mitos constituyen lo que Lévi-Strauss denominó un "grupo de transformaciones» que contiene las variantes registradas de este mito. Los pueblos amazónicos comparten pues con los quechua de las altas tierras el mito señalado.

\section{Discusión y conclusiones}

Quechuas de las altas tierras de los Andes y ashaninkas del piedemonte oriental de los Andes comparten la representación del cerro habitado por un espíritu que provee a los humanos de presas. No son los únicos. Los desana del Vaupés colombiano a los que hemos hecho referencia aquí como referente presentan una representación similar. Tenemos entonces la figura de un mito que atravesando límites ecológicos y culturales se presenta en ambas áreas siendo adaptado a las particularidades de una población de campesinos agricultores- pastores- ganaderos de un lado y de cazadoresrecolectores por otra; un grupo de transformaciones. Ciertamente en el caso andino la figura del espíritu del cerro no se limita a su relación con el ganado. Los cerros tienen puertas secretas y en su interior se encuentran acumuladas ingentes riquezas que sólo en situaciones muy especiales son entregadas en parte a los humanos (ver Ansión 1988). En el caso de los cazadores recolectores amazónicos la riqueza reviste otras formas, la de un aprovisionamiento adecuado de carne y abalorios utilizados como adornos por las mujeres los que son considerados apreciados en igual medida y esta figura no se presenta en sus representaciones acerca del cerro. En el caso ashaninka, su particular proximidad geográfica a las poblaciones andinas y lo rugoso de su territorio llevan a que las figuras de la montańa sagrada y el felino guardián sean parte central de su sistema simbólico directamente relacionado con su vida cotidiana así como otras sociedades indígenas próximas como los matsiguenga (Rosengren 2004: 75) y yanesha

\begin{tabular}{|l|l|l|l|l|}
\hline $\begin{array}{l}\text { Vai-maxë es el protector } \\
\text { de las presas de caza }\end{array}$ & $\begin{array}{l}\text { Las presas se encuentran } \\
\text { en grandes casas dentro } \\
\text { de los cerros }\end{array}$ & $\begin{array}{l}\text { El chamán en su vuelo } \\
\text { psicotrópico viaja a pedir } \\
\text { presas al espíritu en el } \\
\text { cerro }\end{array}$ & $\begin{array}{l}\text { Las presas son soltadas } \\
\text { por vai-maxe y enviadas } \\
\text { a los territorios de caza } \\
\text { de los humanos }\end{array}$ & $\begin{array}{l}\text { El jaguar es el protector } \\
\text { de las presas de caza y } \\
\text { ataca a quien caza más de } \\
\text { lo que necesita }\end{array}$ \\
\hline $\begin{array}{l}\text { Los iriri y la dueńa del } \\
\text { pecari habitan en los } \\
\text { cerros }\end{array}$ & $\begin{array}{l}\text { Las presas están } \\
\text { encerradas en corrales } \\
\text { al interior de los cerros } \\
\text { como si fueran ganado }\end{array}$ & $\begin{array}{l}\text { El chamán viaja } \\
\text { convertido en colibrí } \\
\text { a pedir presas a los } \\
\text { espiritus dueños de los } \\
\text { animales }\end{array}$ & $\begin{array}{l}\text { Las presas son } \\
\text { transportadas por el } \\
\text { chamán convertido en } \\
\text { colibrí en su morral }\end{array}$ & $\begin{array}{l}\text { El jaguar ataca al que } \\
\text { caza más allá de lo que } \\
\text { necesita para subsistir }\end{array}$ \\
\hline $\begin{array}{l}\text { Los espíritus aukis } \\
\text { habitan en los cerros }\end{array}$ & $\begin{array}{l}\text { Los venados son el } \\
\text { ganado de los espíritus } \\
\text { que habitan los cerros }\end{array}$ & $\begin{array}{l}\text { El kintu es enterrado en } \\
\text { el cerro para beneficiar a } \\
\text { la comunidad }\end{array}$ & $\begin{array}{l}\text { Ovejas y auquénidos } \\
\text { bebé emergen de los ojos } \\
\text { de agua del cerro y son } \\
\text { cuidados por el pastor }\end{array}$ & $\begin{array}{l}\text { El puma se lleva el } \\
\text { ganado del que habla mal } \\
\text { de lospíritus del cerro }\end{array}$ \\
\hline
\end{tabular}


(Santos Granero 2004: 315) cercanamente emparentados con los ashaninka. Otros grupos que no cuentan con cerros en sus territorios presentan igualmente la figura del dueño de los animales como ya hemos señalado. Resalta entre estos el caso de los mai-huna en que la divinidad femenina tuara-ako protege a pecaríes y tapires y la divinidad masculina Aima-hiyotu se desplaza por la selva acompañando a sus animales como lo haría un pastor con su hato, maldiciendo a aquellos cazadores que los hieren sin matarlos malgastando así estos recursos escasos (Bellier 1994: 134).

Si hemos privilegiado aquí la figura de la sociedad ashaninka para una comparación con las representaciones de la población quechua andina es porque esta presenta una larga historia de contactos con esta última, tanto positivos como negativos documentados por el registro arqueológico e histórico. Podemos decir que entre los ashaninka la figura del cerro como habitación del dueño de los animales y las presas se presenta mas acentuada que en cualquier otra en la Amazonia, al presentarlas como habitando dentro de corrales análogos a los de los pastores andinos. De otra parte, Wai-max$c \ddot{e}$ entre los desana, iriri y la dueña del pecarí entre los ashaninka y los aukis entre los quechuas presentan las mismas funciones presentándose entre ellos una figura de desdoblamiento. Siempre en el plano mitológico, el jaguar de las bajas tierras y el puma entre los quechua de la altas tierras presentan la misma función de guardianes de las presas y los animales domésticos : en las bajas tierras son castigadores de los cazadores que se exceden en el número de piezas cobradas y en las altas tierras castigan a quienes hablen mal de los espíritus de la tierra y el cerro. Las informaciones etnográficas presentadas nos permiten decir que el caso andino forma parte de un grupo de trasformaciones junto con algunos casos amazónicos.

\section{Agradecimientos}

El presente artículo fue desarrollado al interior del proyecto "Simbología de las montańas sagradas de los Andes» dirigido por Sabino Arroyo Aguilar en la Escuela de Antropología de la Facultad de Ciencias Sociales de la Universidad Nacional Mayor de San Marcos. El autor desea agradecer las referencias bibliográficas brindadas por Teodomiro Palomino Meneses sobre los quechuas del área andina y el trabajo bibliográfico realizado sobre estas en diferentes bibliotecas por el bachiller Samuel Alberto Rojas Chahuaylacc.

\section{Referencias bibliográficas}

Ansión, Juan (1988). Desde el rincón de los muertos. Lima, GRADE.

Arguedas, José María (2013). Obras completas. Ts VI a XII.

Augé, Marc (1979). Simbolo, función e historia. México, Editorial Grijalbo

Barclay, F. y Santos, F. (1995). Órdenes y desórdenes en la selva central. Historia y economía de un espacio regional. Lima, IFEA, IEP, FLACSO

Barthes, Roland (1985). Mitologias. México, Siglo XXI

BeLlier, Irène (1994). «Los Mai Huna». En: Santos,F. y Barclay, F. Guía etnográfica de la Alta Amazonía Vol. I, pp.1-180 Bodley, Jhon H. (1970). Campa Socio-economic adaptation. $\mathrm{Ph}$ D. Dissertation, University of Oregon

Bodley, John H. (1972). «A transformative movement among the campa indians of eastern Peru» En: Anthropos 67 (Friburg) : 220-228

Bodley, John H. (1973). "Deferred exchange among the campa indians » En : Anthropos 68 (Friburg): 589-596

Bonavia, D. y Ravines, R. (1968). «Villas del horizonte tardío en la Ceja de Selva del Perú. Algunas consideraciones», En: Actas y Memorias del XXXVII Congr. Inter. Amer. Tomo I, pp.153-158

BontÉ, Pierre e Izard, Michel (1992). Dictionnaire de l'etnologie et de l'Anthropologie. Paris PUF.

Benveniste, Émile (1969). Le vocabulaire des institutions indo-européenes. 2 Vols. Paris, Les editions de minuit.

Chaumeil, Jean-Pierre (1994). «Los yagua» En: Santos, F. y Barclay, F. Guía Etnográfica de la Alta Amazonia Vol. I. Quito, FLACSO-IFEA.

DumÉziL, Georges (2000). La religión romaine archaïque. Paris, Payot 700pp.

Fernández, Eduardo (1984). «La muerte del Inca. Dos versiones de un mito ashaninka», Anthropológica (Lima) No201-208

FernándeZ, Eduardo (1987). «Los ashaninka y los incas. Historia y mitos», Anthropológica (Lima) no5:335-356

Goulard, Jean-Pierre (2009). Entre mortales e inmortales. El ser según los Tikuna de la Amazonía. Lima, CAAAPIFEA. 458 pp.

Greimas, A.J. y Courtes,J. (1982). Semiótica. Diccionario razonado de la teoría del lenguaje. Madrid, Editorial Gredos.

Havalkof, Soren y Veber, Hanne (2005). «Los Ashéninka del Gran Pajonal» En: Santos Granero,F. y Barclay, F. Guia etnográfica de la Alta Amazonia Vol. V. Smithsonian Tropical Research Institute -Instituto Francés de Estudios Andinos, pp.75-279. 
Lathrap, Donald (1970). The upper Amazon. London, Thames and Hudson.

Lenclud, G. (1992). «Symbolisme» En: Bonté,P. y Izard,M. LÉvi-Strauss, Claude (1958) Anthropologie Structurale. Paris, Plon.

LÉvi-Strauss, Claude (1991). Histoire de Lynx. Paris, Plon

MÉTraux, Alfred (1962). Les Inkas. Paris, Éditions de Seuil, 292pp.

Noble Соок, K. (1965). Proto-Arawakan and its descendants. Mouton, Le Hague. 129 pp

Morin, Françoise (1998). «Los Shipibo-Conibo». E: Santos, F. y Barclay, F. Guia etnográfica de la Alta Amazonía. Quito, Smithsonian Tropical Research Institute- ediciones Abya-Yala, pp. 275-435.

Parsons, J. R. y Hastings, Ch. (1992). "The late intermediate period». En: Keatinge,R.W. (ed.) Peruvian Prehistory, Cambridge University Press, pp. 190-229.

RAYMOND, Scott (1982). «Quimpiri, a ceramic style from the peruvian montaña», Naupa Pacha (Berkeley) no 20.

RaYMOND, Scott (1992). «Highland and colonization of the peruvian montaña in relation to the political economy of the Huari empire», Journal of the Steward Anthropological Society, Vol. 20, no 1-2, pp. 17-36

Renard-Casevitz, F.M., Saignes, Th., Taylor, A.C. (1986). L'Inca, l'Espagnol et les sauvages. Rapports entre les sociétés amazoniennes et andins du XV au XVII siècle. Paris, Editions Recherche sur les Civilisations, pp. 411.

Renard-Casevitz, France-Marie (1992). «Historia Kampa, memoria ashaninka. En: Carneiro da Cunha, M. História dos Indos no Brasil. Sáo Paulo, Editora Schuarcz Ltda, pp. 197-212

Reichel-Dolmatoff, Gerardo (1968). Desana. Simbolismo de los Indios del Vaupés. Bogotá, Universidad de los Andes.

Reichel-Dolmatoff, Gerardo (1970). El chamán y el jaguar. México, Siglo XXI.

Rojas Zolezzi, Enrique Carlos (1994). Los ashaninka un pueblo tras el bosque. Lima, Pontificia Universidad Católica del Perú. 360 pp.

Rojas Zolezzi, Enrique Carlos (2004). «Divinidades objeto en las sociedades amazónicas». En: Marzal,M. Romero,C. y Sánchez, J. Para entender la religión en el Perú. Lima, Pontificia Universidad Católica del Perú, pp. 429-473.

Rojas Zolezzi, Enrique Carlos (2004). Mythes de la création du monde, représentations du gibier et des plantes cultivées et définition de l'ordre social traditionnel chez les campa ashaninka de l'orient péruvien. Deux Vols. 972 pp.

Rojas Zolezzi, Enrique Carlos (2014). El morral del colibrí. Mitología, chamanismo y ecología simbólica entre los ashaninka del oriente peruano. Lima, Editorial Horizonte.
Rosengren, Dan (2004). «Los Matsiguenka». En: Guía etnográfica de la Alta Amazonía Vol. IV. Smithsonian Tropical Research Institute- Instituto Frances de Estudios Andinos.

Santos Granero, Fernando (1988). «Templos y herrerías : utopía y recreación cultural en la Amazonia peruana» (siglos XVIII-XIX)» Bull. Inst. Fr. Et. And. Nro.2:1-22

Santos Granero, Fernando (2004). «Los Yanesha». En: Guia Etnográfica de la Alta Amazonía Vol. IV Smithsonian Tropical Research Institute-Instituto Francés de Estudios Andinos

Sperber, Dan (1971): «El estructuralismo en antropología» En: Ducrot, O., Todorov, T., Sperber, D. Safouan, M. Wahl, F. ¿Qué es el estructuralismo? Buenos Aires, Editorial Losada.

Sperber, Dan (1974). Le symbolisme en general. Paris, Hermann.

Surrallés, Alexandre (2007). «Los Candoshi». En: Santos, F. y Barclay, F. Guía Etnográfica de la Alta Amazonia Vol. VI Smithsonian Tropical Research Institute- Instituto Frances de Estudios Andinos, pp. 247-380.

Tambiah, Stanley (1990). Magic, science, religion and the scope of rationality. Cambridge U.P., New York.

Tibesar, A. Fr. OFM (1950). "The salt trade among the montaña indians of the montaña area of eastern Peru», Primitive Man (Washington) Vol. XXIII:103-108

Tibesar, A. Fr. OFM (1952). «San Antonio de Eneno: a mission in the peruvian montaña", Primitive Man (Washington) Vol. XXV: 23-39.

Urton, Gary (1985). "Animal metaphors and the life cycle in an andean comunity». En: Urton, Gary (ed) Animal myths and metaphors in South America. University of Utah Press.

Valderrama, Ricardo y Escalante, Carmen (1988). Del tata mallku a la mama pacha. Riego, sociedad y ritos en los Andes peruanos. Lima, DESCO.

Valderrama, Ricardo y Escalante, Carmen (1997). La doncella sacrificada. Mitos del valle del Colca. Lima, Universidad Nacional de San Agustín- IFEA.

VARESe, Stefano (1973). La sal de los cerros. Lima, Retablo de papel ediciones. $400 \mathrm{pp}$.

WeIss, Gerald (1975). Campa Cosmology. The world of a forest tribe in South America, Anthropological Papers of the Museum of Natural History, New York.

Weiss, Gerald (2005). «Campa Ribereños». En: Santos Granero, F. y Barclay, F. Guia Etnográfica de la Alta Amazonía Vol. V. pp. 1-7. 